\title{
Measuring of knee cartilage thickness: A comparison between ultrasound and magnetic resonance imaging methods
}

\author{
Lida Shashaani ${ }^{*}$, Armin Asiachi ${ }^{2}$, Omid Motamedi ${ }^{1}$ \\ ${ }^{1}$ Hzrat e Rasool Hospital, Iran University of Medical Sciences, Tehran, Iran. ${ }^{2}$ Alborz University of Medical Sciences, Karaj, Iran.
}

Cartilage diameter evaluation is critical for cartilage assessment. Magnetic resonance imaging (MRI) is the gold standard tool in cartilage evaluation. This observational and analytical study was designed to answer the question of whether there is a relationship between MRI and ultrasound in measuring cartilage thickness in the medial femoral condyle. The current study was conducted at the Radiology Department of Rasole-Akram Hospital, Tehran, Iran, between March and May 2020. The sample size was 18 people. The mean cartilage thickness of the left medial femoral cartilage was measured by T1 weighted MRI and ultrasound from transverse, anterior, middle, and posterior medial femoral regions in nine healthy females with a mean \pm std deviation as indicated below:thickness $=1.6 \pm 0.04 \mathrm{~m}$, weight $=55.3 \pm 4.3 \mathrm{~kg}$, age $=21.7 \pm 0.8$ years.

Additionally, nine healthy males with thickness $=1.80 \pm 0.02 \mathrm{~m}$, weight $=78.6 \pm 11.1 \mathrm{~kg}$, age $=22.4 \pm 0.7$ years were also included. Pearson and Bland-Altman plots were used for correlations and agreements. Anterior longitudinal ultrasound thickness measures were significantly positively correlated with MRI anterior $(\mathrm{r}=0.93, p=0.00001)$, transverse ultrasound with MRI anterior $(\mathrm{r}=0.87 p=0.0369)$, middle longitudinal ultrasound with MRI anterior $(\mathrm{r}=0.87 p=0.00002)$, and transverse ultrasound and MRI middle $(\mathrm{r}=0.87 p=0.00001)$.

Agreement in all aspects was good except between the anterior longitudinal ultrasound and MRI posterior. There was good absolute agreement between corresponding measurements done by ultrasound and MRI. The results suggest that ultrasound may be a good clinical tool for assessing relative cartilage thickness in medial femoral regions.

Keywords: osteoarthritis, medial femur, clinical evaluation, cartilage thickness

\section{Introduction}

The evaluation of cartilage thickness is mandatory in many instances, such as knee osteoarthritis (OA). Cartilage thickness reflects both the health condition of the cartilage and the treatment effectiveness. Hence, an accurate and easy-to-use technique for evaluating cartilage thickness is necessary.

An accurate measurement of cartilage thickness helps to detect and monitor cartilage condition; thus, effective measurement tools are needed to detect both increases and decreases in medial femoral cartilage thickness.

MRI is an important assessing tool in detecting both OA onset and progression [1, 2]. It is known that during the early stage of OA, there might be an increase in cartilage thickness [3, 4]. Individuals with established knee OA have thinner tibio-femoral cartilage thickness compared to healthy subjects [5]. It has been suggested that assessing central medial femoral cartilage for changes in morphology and diameter is helpful in detecting early signs of knee OA [6].

MRI is the gold standard for evaluating knee cartilage thickness [7]. MRI is expensive, not available for all patients all the time, and not easily available for serial evaluation of cartilage status. Conventional radiography is more available, but it reveals only gross joint space narrowing [8]. Ultrasound offers an alternative quantitative measurement of cartilage thickness which is more available and more cost-effective compared to MRI [9]. Ultrasound is easy to use, low cost, and more available. It is available to evaluate the status of the femoral cartilage in the OA population $[10,11]$.

If ultrasound were found to be a valid and reliable measurement tool for cartilage thickness in uninjured or healthy knees, it would be promising news. The first step in the current study was to find an association and absolute agreements between ultrasound and MRI in measuring

Personal non-commercial use only. Rheumatology Research Journal. Copyright (C 2020. All rights reserved

*Corresponding Author: :Lida shashaani, MD Rheumatologist, Hzrat e Rasool hospital, Iran University of Medical Sciences, Tehran, Iran. PO-Box: 2188054188, E-mail lidashashaani@yahoo.com, Telefax: +98-919-768-1929.

Received: 01 August 2019; Accepted: 30 December 2019 
medial femoral condyle cartilage thickness in healthy individuals. It was hypothesized that ultrasound and MRI diameter measurements of medial femoral condyle cartilage thickness would be strongly correlated with each other and would demonstrate a high degree of absolute agreement.

\section{Materials and Methods}

\section{Experimental protocol}

The current study was conducted in Tehran, Iran, between March and May 2019. Based on a similar study, the sample size was 18 people, and evaluations were done in the Radiology Department of Rasole-Akram Hospital (Tehran)

Nine healthy females (mean \pm std dev) $(1.6 \pm 0.04 \mathrm{~m}$, $55.3 \pm 4.3 \mathrm{~kg}, 21.7 \pm 0.8$ years $)$ and nine healthy males $(1.80$ $\pm 0.02 \mathrm{~m}, 78.6 \pm 11.1 \mathrm{~kg}, 22.4 \pm 0.7$ years) participated in this cross-sectional observational analytical study. The university's Institutional Review Board approved the study, and all participants gave written informed consent. Eligibility criteria were age younger than 25 years, BMI less than 30 , no current or past orthopedic injury or history of significant injury or surgery in the left limb, no recent or past history of thyroid disease, no recent or past history of parathyroid disease, no alignment problems such as genu varus or genu valgus, and no autoimmune or connective tissue disease or ligament laxity. Participants were selected in random order and then MRIs were done. Thereafter, ultrasound measuring was done. Cartilage thickness was measured with ultrasound and MRI in separate sessions. Both sessions were conducted at the same time of day $( \pm 1 \mathrm{~h})$ in order to account for possible diurnal effects of cartilage physiology. The assessments were completed in 30 days.

\section{Procedures}

\section{Ultrasound measurement of cartilage thickness}

A single examiner performed all ultrasound imaging of the medial femoral articular cartilage using a previously established transverse [12] and longitudinal [13] approach. All ultrasound assessments were performed on the left knee with a $10 \mathrm{MHz}$ linear ultrasound transducer (Philips, Inc. Affinity 70G, Netherlands).

For the transverse approach, following 10 minutes of quiet sitting, participants were instructed to sit on a table with their left knee placed at $90^{\circ}$ of flexion. The bony medial femoral condyle anatomy was palpated, and the bony anatomy was outlined with a washable marker. The transducer head was then placed transversely over the medial femoral condyle to measure cartilage thickness over the mid-portion of the medial femoral condyle (most weight-bearing portion). Three adjacent $(\sim 2 \mathrm{~mm})$ cartilage thicknesses were measured from a thin hyper-echoic line at the soft tissue-cartilage interface to the hyper-echoic line at the cartilage-bone interface. These three values were averaged to comprise a single thickness value, which was representative of the middle medial femoral condyle cartilage thickness.

For the longitudinal approach, patients were placed in a seated position on a table with the knee in maximal flexion $\left(133^{\circ}\right.$ to $\left.151^{\circ}\right)$. The transducer head was then placed longitudinally in the sagittal plane midway between the medial border of the patella and medial border of the medial femoral condyle. Cartilage thickness was assessed over the anterior, middle, and posterior regions of the medial femoral condyle. At each region, three adjacent $(\sim 2$ $\mathrm{mm}$ ) cartilage thicknesses were measured from a thin hyper-echoic line at the soft tissue-cartilage interface to the hyper-echoic line at the cartilage-bone interface (Figure 1). These three values were averaged to achieve a single value that was representative of each region's cartilage thickness.

\section{MRI measurement of cartilage thickness}

MRI examination of the left knee was obtained with a 1.5 T MRI system (Philips, Ingenia, Nederland) using a 16channel transmit/receive high-resolution knee coil. Articular cartilage measurements were acquired with 3D Fast Field Echo sagittal MRI scans of the tibio-femoral joint (slice thickness $=1 \mathrm{~mm}$, in-plane resolution $=0.7 \mathrm{~mm}$ $\times 0.7 \mathrm{~mm}$, number of slices $=167$, image matrix $=227 \times$ $228, \mathrm{TR}=32 \mathrm{~ms}, \mathrm{TE}=14 \mathrm{~ms}$, flip angle deg $=40$, bandwidth $(\mathrm{Hz} /$ pixel $)=623.0 \mathrm{~Hz}$, fat suppression $=$ preset.

Following an MRI data acquisition, a single investigator manually segmented the articular cartilage of the medial femoral condyle. Following the methods of Koo et al. [16], the medial femoral condyle was segmented into three regions of interest (anterior, middle, and posterior) that estimate the main weight-bearing regions of the femoral articular cartilage. In order to determine the regions of interest, first a central axis perpendicular to the sagittal plane was located using a cylinder fitting method that fit the medial femoral cartilage surface geometry of the sagittal slice representing the midpoint of the medial femoral condyle. Next, a line was drawn from the central medial tibio-femoral contact point to the cylinder center. The anterior region was defined as 0 to $30^{\circ}$ anterior to this line, the middle region was defined as 0 to $30^{\circ}$ posterior to this line, and the posterior region was defined as 30 to $60^{\circ}$ posterior to this line. Finally, the weight-bearing region width was defined as $20 \%$ of the overall maximal medial to lateral condylar width centered on the midline of the medial condyle. For each region, pixel-wise thickness maps were created in ImageJ (https://imagej.nih.gov/ij/) by calculating the diameter of the largest sphere that fit inside the segmented region and contained the point as the sphere center. The average thickness over each of the three regions was then calculated for inclusion in the analyses. 


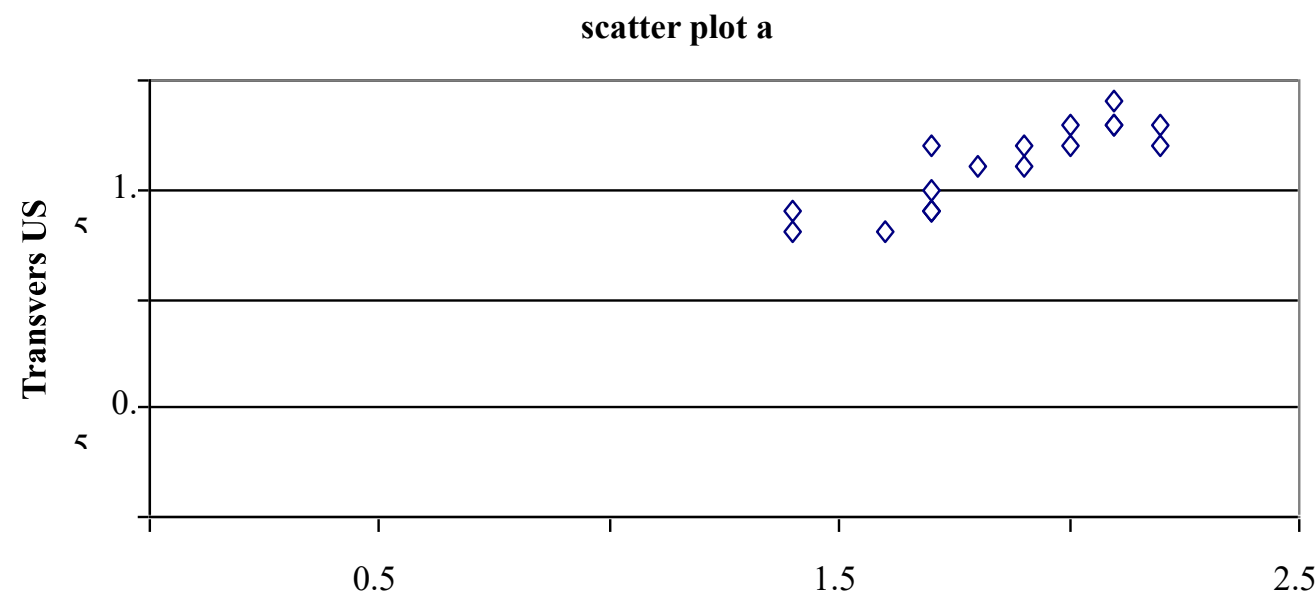

Series1

Mid-femoral MRI

scatter plot b

Series1

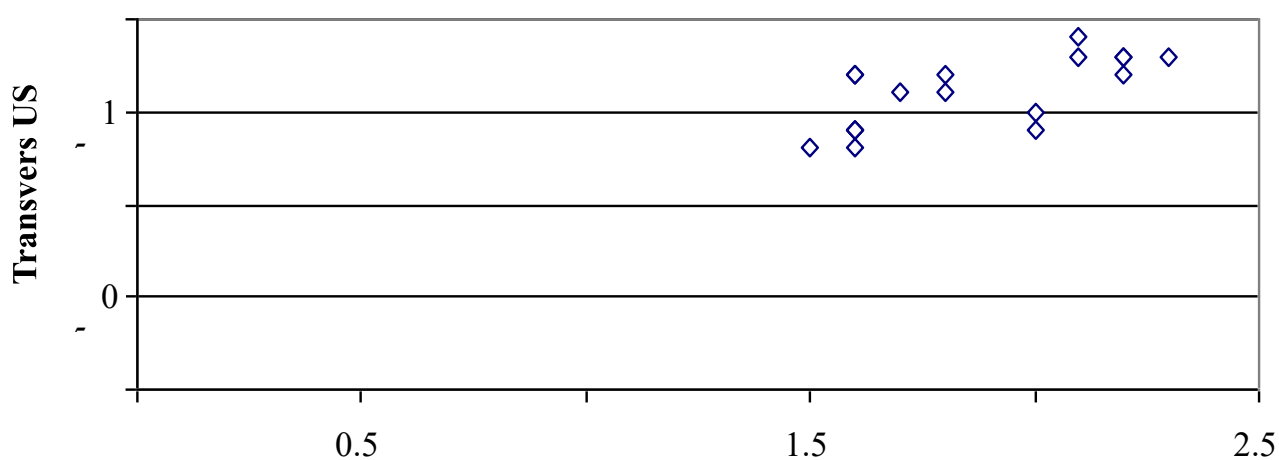

Post-femoral MRI

scatter plot c

Series1

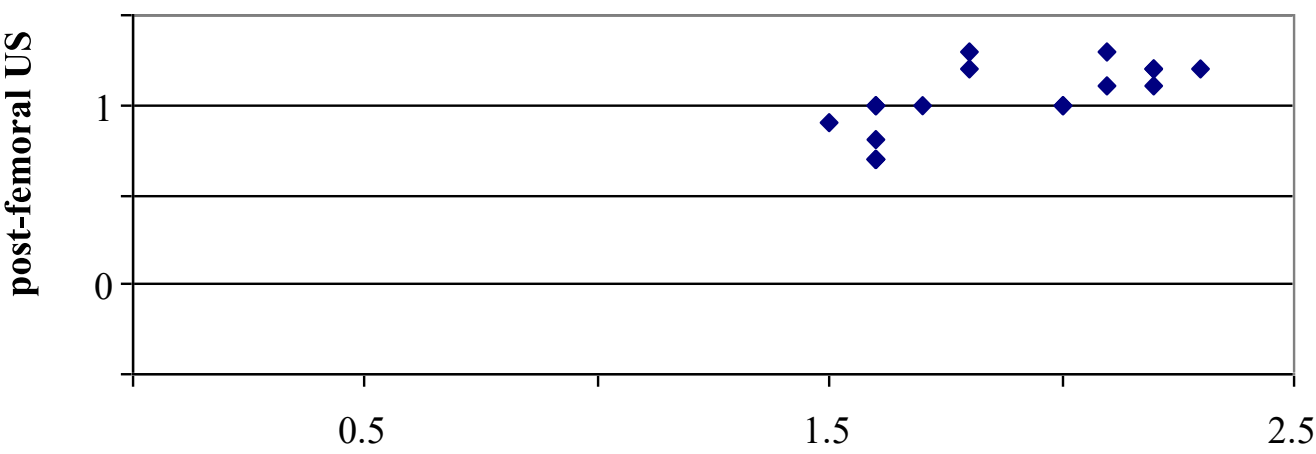

Post-femoral MRI

Figure 1: Scatter plots of (a) transverse ultrasound to mid-femoral MRI thicknesses; (b) transverse ultrasound to posterior femoral MRI thicknesses; (c) posterior femoral ultrasound to posterior femoral MRI thicknesses. 


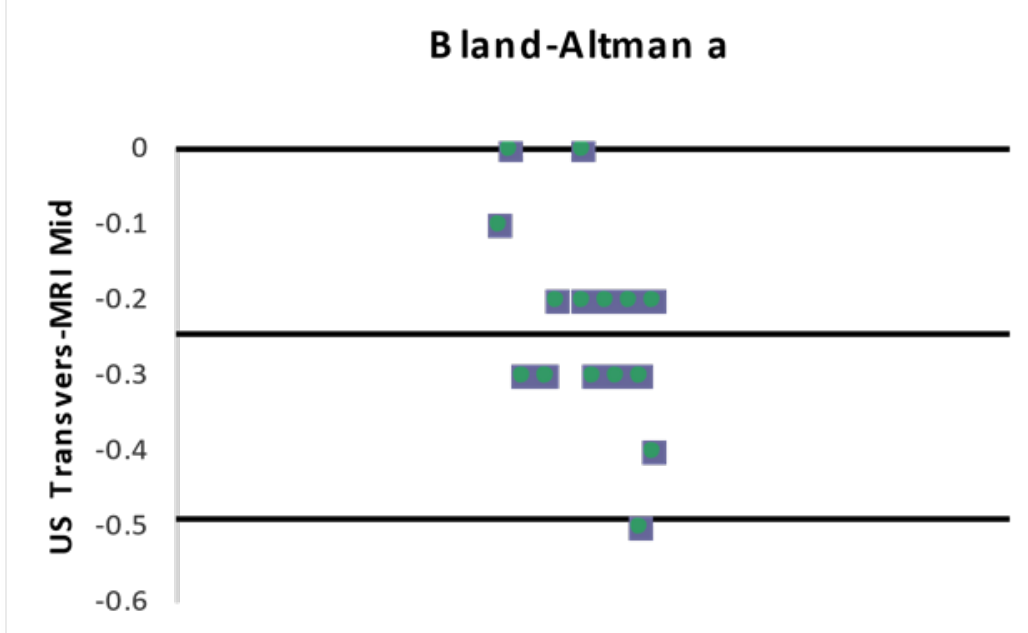

Mean

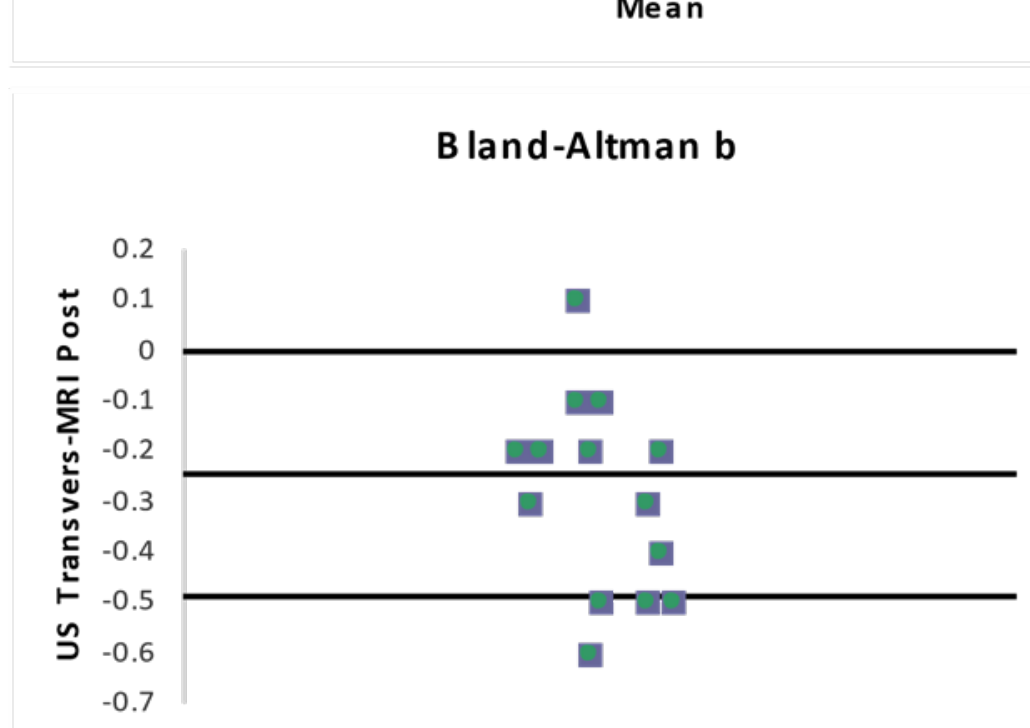

Mean

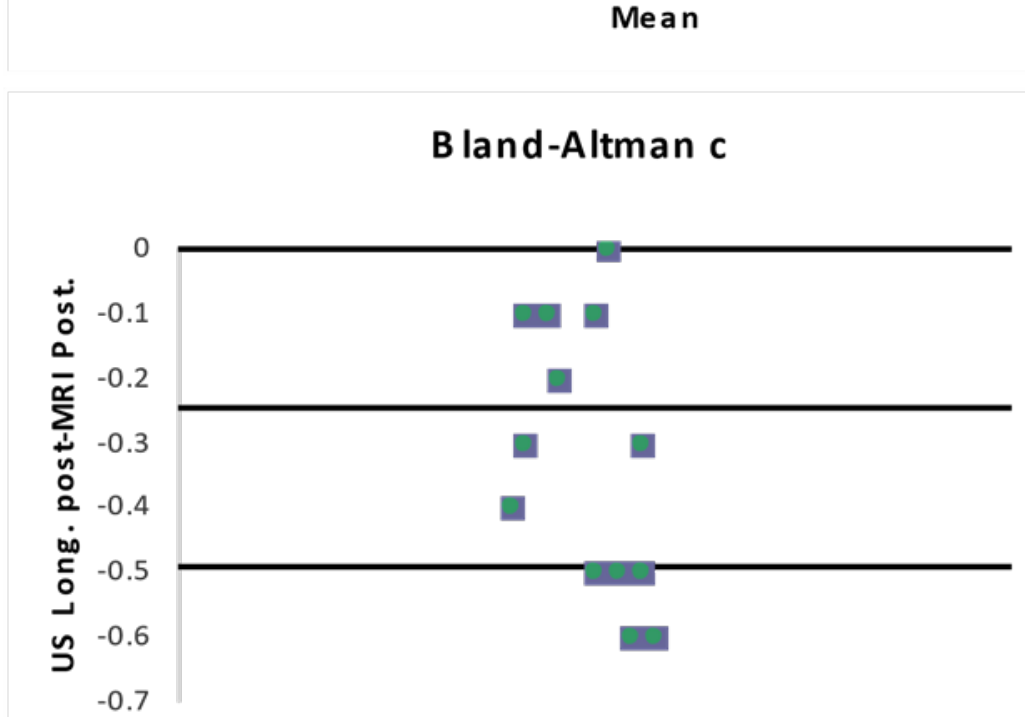

Mean

Figure 2: Bland-Altman plots to illustrate the agreement between (a) transverse ultrasound and mid-femoral MRI thickness; (b) transverse ultrasound and pos- terior femoral MRI thickness; (c) longitudinal posterior femoral ultrasound and pos- terior femoral MRI thickness. 


\section{Results}

Descriptive data by gender is presented in Table 1 . Descriptively, there were main effects $(p \leq 0.05)$ for gender and imaging modality with female and ultrasound measurements resulting in decreased thicknesses, respectively (Table 1).

All ultrasound thickness measures were significantly smaller than MRI measures $(p$ value $=0.00075)$. Females had a significantly smaller overall thickness than males ( $p$ value $=0.00253)$. Anterior MRI thickness was significantly smaller than anterior and middle MRI thicknesses ( $p$ value $=0.033)$.

For the main research questions, the magnitudes of associations are presented in Table 2.
Anterior longitudinal ultrasound thickness measures were significantly positively correlated with MRI anterior $(\mathrm{r}=0.93, p$ value $=0.00001)$, transverse ultrasound with MRI anterior $(\mathrm{r}=0.87, p$ value $=0.0369)$, middle longitudinal ultrasound with MRI anterior $(\mathrm{r}=0.87, p$ value $=0.00002)$, and transverse ultrasound and MRI middle $(\mathrm{r}=0.87, p$ value $=0.00001)$.

The correlation between anterior longitudinal ultrasound and MRI posterior was weak $(\mathrm{r}=0.49, p$ value $=0.00008)$.

Agreement was good in all aspects except the anterior longitudinal ultrasound and MRI posterior, in which agreement was weak.

Bland-Altman plots demonstrated good absolute agreement between correlated measures with ultrasound and MRI.

Table 1.Descriptive outcomes (mean \pm SD) of MRI and ultrasound measures of cartilage thickness.

\begin{tabular}{|c|c|c|c|c|c|c|c|}
\hline \multirow{2}{*}{$\begin{array}{l}\text { Medial femoral cartilage } \\
\text { regions }\end{array}$} & \multicolumn{2}{|c|}{ Combined } & \multicolumn{3}{|c|}{ Females $^{\dagger}$} & \multicolumn{2}{|l|}{ Males } \\
\hline & & $\begin{array}{l}\text { MRI } \\
\text { ckness } \\
\text { mm) }\end{array}$ & $\begin{array}{c}\text { US } \\
\text { thickness * } \\
\text { (mm) }\end{array}$ & $\begin{array}{c}\text { MRI thickness } \\
\text { (mm) }\end{array}$ & $\begin{array}{c}\text { US } \\
\text { thickness } \\
\text { (mm) }\end{array}$ & $\begin{array}{c}\text { MRI } \\
\text { thickness } \\
(\mathbf{m m})\end{array}$ & $\begin{array}{c}\text { US } \\
\text { thickness } \\
\text { (mm) }\end{array}$ \\
\hline Anterior & 1.7 & $\pm 0.3 * *$ & $1.5 \pm 0.3$ & $1.5 \pm 0.2$ & $1.3 \pm 0.2$ & $1.7 \pm 0.1$ & $1.7 \pm 0.1$ \\
\hline Middle & 1.8 & \pm 0.2 & $1.6 \pm 0.3$ & $1.7 \pm 0.2$ & $1.3 \pm 0.1$ & $1.8 \pm 0.1$ & $2 \pm 0.1$ \\
\hline Posterior & 1.9 & \pm 0.2 & $1.7 \pm 0.2$ & $1.7 \pm 0.2$ & $1.4 \pm 0.1$ & $1.8 \pm 0.1$ & $2 \pm 0.1$ \\
\hline Transverse & & & $1.6 \pm 0.2$ & & $1.5 \pm 0.1$ & & $1.7 \pm 0.1$ \\
\hline
\end{tabular}

*All ultrasound thickness measures significantly smaller than MRI measures, $P$ value $=0.00075$ anterior MRI thickness was significantly smaller than anterior and middle MRI thicknesses. $P$ value $=0.033$

${ }^{\dagger}$ Female had significantly smaller thickness than mal. $P$ value $=0.00253$

Table 2. Articular cartilage thickness Pearson correlation r values.

\begin{tabular}{|c|c|c|c|c|c|c|c|}
\hline $\begin{array}{l}\text { Thickness } \\
\text { variable }\end{array}$ & US-Trans & US Ant-Long & US Mid-Long & US Post-Long & MRI-Ant & MRI-Mid & MRI-Post \\
\hline US-Trans & 1 & $0.82 * \mathrm{p}=0.045$ & $0.85 * \mathrm{p}=0.043$ & $0.73 * p=0.034$ & $\begin{array}{c}0.87 \\
* p=0.036\end{array}$ & $\begin{array}{c}0.87 \\
* p<0.001\end{array}$ & $\begin{array}{c}0.68 \\
*_{p}<0.001\end{array}$ \\
\hline US Ant-Long & & 1 & 0.86 & 0.74 & $\begin{array}{c}0.93 \\
*_{p}<0.001\end{array}$ & $\begin{array}{c}0.79 \\
* \mathrm{p}<0.001\end{array}$ & $\begin{array}{c}0.49 \\
* p<0.001\end{array}$ \\
\hline US Mid-Long & & & & 0.79 & $\begin{array}{c}0.87 \\
*_{p}<0.001\end{array}$ & $\begin{aligned} 0.85 \\
* \mathrm{p}<0.001\end{aligned}$ & $\begin{array}{c}0.72 \\
* p<0.001\end{array}$ \\
\hline US Post-Long & & & & 1 & $\begin{array}{c}0.76 \\
*_{p}=0.003\end{array}$ & $\begin{array}{c}0.74 \\
* \mathrm{p}<0.001\end{array}$ & $\begin{array}{c}0.7 \\
* \mathrm{p}<0.001\end{array}$ \\
\hline MRI-Ant & & & & & 1 & 0.75 & 0.5 \\
\hline MRI-Mid & & & & & & 1 & 0.77 \\
\hline MRI-Post & & & & & & & 1 \\
\hline
\end{tabular}

\section{Discussion}

There is a need for easily accessible and cost-effective clinical tools to assess the health of knee articular cartilage. Thus, this study was designed to better understand how well clinical ultrasound thickness measures compared with the gold standard MRI measures. The primary finding was that the transverse, anterior mid-longitudinal, and posterior longitudinal ultrasound measures were more strongly correlated with those from the anterior, middle, and 
posterior MRI regions. The absolute agreement between these measures was relatively good. The following discussion will address the potential use of ultrasound as a tool to assess knee cartilage thicknesses as well as the potential reasons for the outcomes to be associated with poor absolute agreement. Longitudinal ultrasound measures were moderately $(r=0.57)$ and strongly $(r=0.84)$ correlated, respectively, with MRI-based measures in an OA population [13].

Additionally, femoral condyle articular cartilage thicknesses were strongly correlated (rho values $=0.71$ to 0.86) with MRI measurements in children with juvenile idiopathic arthritis [9]. Ultrasound has also demonstrated good agreement with the physical measurement of cartilage thickness in the medial femoral condyle of older cadaver knees $(\mathrm{ICC}=0.72)$ [12].

It is likely that the primary weight-bearing regions selected for MRI analyses in the current study were more posteriorly located relative to the placement of the transducer in the anterior longitudinal ultrasound measure; thus, the different measures between ultrasound and MRI may be due to positional limitations.

The findings of smaller ultrasound measurements in comparison with MRI measurements at the knee were reported in a range of healthy children, where the anterior and posterior cartilage thicknesses were 0.7 and $0.9 \mathrm{~mm}$ smaller, respectively, when using ultrasound compared to MRI [14]. Spannow et al. reported similar observations [15].

As an explanation for the smaller ultrasound measures, it was suggested that ultrasound may underestimate cartilage thickness compared to MRI due to the positioning of the ultrasound transducer on a small fluid-covered surface necessary for transduction, which may result in obliquity [14]. Additionally, it has been hypothesized that ultrasound may underestimate the actual cartilage thickness values because of the more rapid sound transmission speed in cartilage than in all soft tissue [8]. Thus, we suggest that ultrasound-based measures may be a relative indicator of thickness but not an absolute indicator of actual cartilage thickness.

When seeking to assess the validity of any measure, it is important to compare the current values to those previously obtained by others. Analyzing MRI-based cartilage thicknesses of the same regions as the current study showed that average thicknesses ranged from 2.1 to $2.4 \mathrm{~mm}[16]$, whereas in the current study they ranged from 1.67 to $2.04 \mathrm{~mm}$. The current study had a younger population (mean age of 22 years compared with the previously reported age range of 26 to 38 years with mean age reported) as age-related thinning of cartilage occurs as part of the aging process [17]; the previous study assessed only five individuals. A recent study showed condylar cartilage-thickness ranged from 3.6 to $4.3 \mathrm{~mm}$ [1]. This study did not have thicker cartilage measures, possibly because of the 1.5 Tesla machine used, technical error, or racial differences. It is important to note that these imaging modalities, ultrasound and MRI, have their own strengths and weaknesses. Ultrasound is beneficial to the clinician and patient due to its lower cost and greater availability/access; however, while MRI measures are more labor intensive and costly to perform, MRI offers the ability to fully image the entire knee cartilage (ultrasound cannot fully visualize the tibial cartilage). Choice of imaging modality must take these multiple factors into account.

The current study is limited to analyses of the medial femoral condyle. Due to greater clinical incidence of medial knee joint OA (as the ratio of medial to lateral cartilage disease is roughly five to one among women and eight or nine to one among men), another question is whether the same relationships would hold true for the lateral femoral condyle.

Due to joint restrictions and bony anatomy, it is impossible to obtain valid measurements of tibial articular cartilage.

\section{Conclusion}

There were moderate to strong correlations between MRI and ultrasound measures of cartilage thickness in the medial femoral condyle; however, ultrasound may underestimate cartilage thickness relative to MRI. Ultrasound may be a useful clinical tool for assessing cartilage thickness in serial investigations, although this needs to be demonstrated in follow-up studies.

\section{Funding}

This research did not receive any specific grant.

\section{Acknowledgements}

The authors deeply thank Dr. anoshe haghighi for her

support and help.

\section{Conflict of interest}

None 


\section{References}

1. Randy J, Schmitz a, Hsin-Min Wang b, Daniel R, Polprasert a, Robert A. et al. Pietrosimone Evaluation of knee cartilage thickness: A comparison between ultrasound and magnetic resonance imaging methods. Knee 2016; 24(2):217-23. doi: 10.1016/j.knee.2016.10.004.

2. Eckstein F, Cicuttini F, Raynauld JP, Waterton JC, Peterfy C. Magnetic resonance imaging (MRI) of articular cartilage in knee osteoarthritis (OA): morphological assessment. Osteoarthritis Cartilage 2006; 14(Suppl. A): A46-75. doi: 10.1016/j.joca.2006.02.026.

3. BuckRJ, Wyman BT, Le Graverand MP, Hudelmaier M, Wirth W, Eckstein F. et al. Osteoarthritis may not be a oneway-road of cartilage loss - comparison of spatial patterns of cartilage change between osteoarthritic and healthy knees. Osteoarthritis Cartilage 2010; 18(3):32935. doi: 10.1016/j.joca.2009.11.009.

4. Frobell RB, Nevitt MC, Hudelmaier M, Wirth W, Wyman BT, Benichou O. et al. Femorotibial subchondral bone area and regional cartilage thickness: a cross-sectional description in healthy reference cases and various radiographic stages of osteoarthritis in 1003 knees from the Osteoarthritis Initiative. Arthritis Care Res (Hoboken) 2010; 62(11):1612-23. doi: 10.1002/acr.20262.

5. Cicuttini FM, Wluka AE, Stuckey SL. Tibial and femoral cartilage changes in knee osteoarthritis. Ann Rheum Dis 2001; 60(10):977-80. doi: 10.1136/ard.60.10.977.

6. Frobell RB, Le Graverand MP, Buck R, Roos EM, Roos HP, Tamez-Pena J. et al. The acutely ACL injured knee assessed by MRI: changes in joint fluid, bone marrow lesions, and cartilage during the first year. Osteoarthritis Cartilage 2009; 17(2):161-67. doi: 10.1016/j.joca.2008.06.020.

7. Stammberger T, Eckstein F, Englmeier KH, Reiser M. Determination of 3D cartilage thickness data from MR imaging: computational method and reproducibility in the living. Magn Reson Med 1999; 41(3):529-36. doi: 10.1002/(sici)1522-2594(199903)41:3<529::aidmrm15>3.0.co;2-z.

8. Pradsgaard DO, Fiirgaard B, Spannow AH, Heuck C, Herlin T. Cartilage thickness of the knee joint in juvenile idiopathic arthritis: comparative assessment by ultrasonography and magnetic resonance imaging. $\boldsymbol{J}$ Rheumatol 2015; 42(3):534-40. doi: 10.3899/jrheum.140162.
9. Ostergaard M, Court-Payen M, Gideon P, Wieslander S, Cortsen M, Lorenzen I. et al. Ultrasonography in arthritis of the knee. A comparison with MR imaging. Acta Radiol 1995; 36(1):19-26.

10. Keen HI, Wakefield RJ, Conaghan PG. A systematic review of ultrasonography in osteoarthritis. Ann Rheum Dis 2009; 68(5):611-19. doi: 10.1136/ard.2008.102434.

11. Abraham AM, Goff I, Pearce MS, Francis RM, Birrell F. Reliability and validity of ultrasound imaging of features of knee osteoarthritis in the community. BMC Musculoskelet Disord 2011; 12:70. doi: 10.1186/14712474-12-70.

12. Naredo E, Acebes C, Moller I, Canillas F, de Agustin JJ, de Miguel E, et al. Ultrasound validity in the measurement of knee cartilage thickness. Ann Rheum Dis 2009; 68(8):1322-27. doi: 10.1136/ard.2008.090738.

13. Yoon CH, Kim HS, Ju JH, Jee WH, Park SH, Kim HY. Validity of the sonographic longitudinal sagittal image for assessment of the cartilage thickness in the knee osteoarthritis. Clin Rheumatol 2008; 27(12):1507-16. doi: 10.1007/s10067-008-0956-3.

14. Keshava SN, Gibikote SV, Mohanta A, Poonnoose P, Rayner T, Hilliard P. et al. Ultrasound and magnetic resonance imaging of healthy paediatric ankles and knees: a baseline for comparison with haemophilic joints. Haemophilia 2015; 21(3):e210-22. doi: 10.1111/hae.12614.

15. Spannow AH, Stenboeg E, Pfeiffer-Jensen M, Fiirgaard B, Haislund M, Ostergaard M. et al. Ultrasound and MRI measurements of joint cartilage in healthy children: a validation study. Ultraschall Med 2011; 32(Suppl. 1):S110-16. doi: 10.1055/s-0029-1245374

16. Kornaat PR, Reeder SB, Koo S, Brittain JH, Yu H, Andriacchi TP. et al. MR imaging of articular cartilage at $1.5 \mathrm{~T}$ and $3.0 \mathrm{~T}$ : comparison of SPGR and SSFP sequences. Osteoarthritis Cartilage 2005; 13(4):338-44. doi: 10.1016/j.joca.2004.12.008.

17. Hudelmaier M, Glaser C, Hohe J, Englmeier KH, Reiser M, Putz R. et al. Age-related changes in the morphology and deformational behavior of knee joint cartilage. Arthritis Rheum 2001; 44(11):2556-61. doi: 10.1002/1529-0131(200111)44:11<2556::aid$\operatorname{art436>3.0.co;2-u.~}$ 
